Einstein and Mythology : The Lengthier the Relations in a Myth the Greater Its' Mass

Marvin E. Kirsh 1

Kirsh2152000@yahoo.com

1 The California State University Los Angeles department of Anthropology 


\title{
Einstein and Mythology : The Lengthier the Relations in a Myth the Greater Its' Mass
}

\begin{abstract}
The theory of relativity (1) is considered form a perspective of folklore. Abstracted entities in the theory of relativity are stripped of units in order to provide explanation, to expose an ordinary meaning that employs a fulcrum for visual description. It is suggested that components of the theory's construction are not only unusually compatible with the religious and spiritual but are also unaccounted for scientifically; they may not render the expected power struggle of church doctrine with scientific notions but an opposite situation in which logical contradiction at the root level of physical meaning and symbolism is absent and might exist only with respect to active perceptual structuring, either functioning on the unknown or belief. This situation, is projected to exist in a volatile mythological form as a 'fulcrum' like bridge between points of dispersion in which the (invisible) entity of mass assumes an added social (or physical) weight imposed by the assumption of the existence of massless space; especially, should its' logically non excludable converse situation, of exclusively "mass and gravitational force containing space" for all phenomenon, find future explanation and validity.
\end{abstract}

\section{Discussion}


If one tries to visualize points of farthest dispersions of myths in attempts to correlate culture with history, hidden within the concept of geographical distance is the more rudimentary concept of distance. One might not avoid to consider lengths in terms of triangulations as he visualizes the transit by word of mouth or by written works the physical paths assumed in the migration of folklore, myths, and tales as they relate to culture. Consider the notion of "concept" itself as in theory and its' meaning. For example one might not find physical correlations for the idea of "color", but for a specific color. One might affirm the existence of 'blue' for instance, and in the same breath cannot dispute the existence of color. but if he tries to image it cannot produce but particulars in example. In the world of mythology language and learning is rendered to familiar distances of relation to topics of meaning; the body of works seem to increase in 'mass' with both temporal and physical distance to proximal relations. If one tries again to visualize entities from the theory of relativity, mass for instance, he can assume only images of colored objects, still unable to refuse the existence of the concept of mass. If one can exclude factors of time, replace them with a notions of distance, (mass also like time, cannot be visualized but for particulars of situations, but for the ones plus ones equals twos-one step per second for two seconds amounts to two steps along the brown earth) he arrives with a relation, analogy from the relation from the special theory of relativity, $\mathrm{E}=\mathrm{mc}^{\wedge} 2$, of weight with respect to distance. On reading or with advanced study one might accept this associative description of the theory of relativity, in full form, a tentatively coherent mathematical rendition of the laws of nature as myth, but it may seem relevant, if not just for the purpose of learning and curiosity to pursue a more tangible visualization to compare it with, in a step by step correspondence, with the abstractions, content and meaning of actual concept of the theory as almost complete and totally conceptual in nature, as none of its components (energy, mass, time, velocity) are tangibly 
conceivable in a single step, much less two or more steps, before entities are visualizable as colored quantities with which to relate. Energy must be reduced to force with time, mass to weight in a gravitational field (e.g. force); but then we have an equation that describes force with respect to force, velocity to physical distance with time, time to change to any example one might conceive of that involves physical comparison, and we arrive at an expression of force over time as a relation of force to its' rate of change with distance; in essence the theory of relativity, as it is commonly told, states that time is relative (e.g force as function of time at a given perspective is related to force as function of some function of time at another.perspective) In reverse if one begins with weight and distance, working backwards, the equations of the theory of relativity proclaim weight as a function of distance, less for, but including, in strict interpretation of the mathematics, an accounting of the 'entityof mass', which one might only visualize as a conceptual variable which renders weight in proximity to other masses. Light, which can only be visualized in terms of color is also given a component of mass because the figures do not add up if one assumes that light possess energy, as energy is stated as the multiplication product of the velocity of light times the quantity of mass. It has also been discovered ( and was predicted from the theory of relativity) that light rays bend in the presence of masses, so that it seems reasonable to attribute this to the interaction as ba gravity from the mass of light with other masses to balance the equation. Theory and test now seek to account for the mass property of light, as mass is apparently a hidden factor to the senses; time is more easily constructed abstractly than mass and it seems absurd also to it to attribute weight, as mass can be visualized to time, but paradoxically in weight reduction classes, based on facts of biochemical metabolism which rest upon knowledge of the physics of energy. It is at this level of individual experience, transmitted notions of self and others, i.e. myth, that the sciences 
intersect conceptually with the self. Practical sensory experience of the world comes to test at this intersection, concept with example, the theory of relativity can make sense abstractly at the same time that it appears as an absurdity, confrontation, to consider a proportionality, time passing relatively one frame to the next other than just passing. The relation, though, of weight proportional to distance is not as hard to construe. Consider a lever, fulcrum with which to move a heavy weight, at the far end the weight is less and it is not so difficult to claim with respect to notions of distance in mythology, that if the weight of a myth seems greater at the distal end, that the most leverage, influence on culture, is not proximal. The point of furthest dispersion of myths, considered to be the most stabile are indeed the most proximal, as one might expect to be the case of a weight on the end of a lever. This distance, the length of a lever, in relativity theory places the first person perspective at one end, distances as vast as light years at the distant end, ideas of length contraction/expansion along an abstractly defined lever with mathematical examples of travel along the lever as time travel; a man parting from the earth at very high speeds and returning can be calculated to have aged less at a distal point where, with ratio, the relative forces applied in order to move either end are made fromcalculation using the formula $\mathrm{E}=\mathrm{Mc}^{\wedge} 2$. On the surface of this conceptualization a visualization may be created as a construction to move the present, change its' age via a mechanism of fast travel via the conceptualization of a weightless entity, mass, from which weight is derived, and light, which possesses scientifically the source of all biological energy, also possesses. Thus one might think to convert light into mass experimentally, move it and recover it, and this has been accomplished, though the intensity of the light is observed to be decreased without change in markers that make it identifiable as the original light (suspect that it exerted change of some form without self change). 
As over and over again throughout history, the potential facts of myth are put to test. Suspect specimens for the original Noahs' Ark are reported, for the places of birth and death of Christ, his remains, etc. though they are ever present conceptually as a weight in the form of an inherited social responsibility to the proximal facets of daily living. Though verification of the material existence of the ancient relics of myths may add additional facts and depth to their story, it bears little of the tangible fact of the existence of the myth and its' propagation. It might seem inappropriate to, though not totally illogical to produce the given constant of the velocity of light in the equation $\mathrm{E}=\mathrm{Mc}^{\wedge} 2$ as 'the mass of the potential energy' of an entity that imposes a mass to society; to seek it out for verification, yet if it is ever present, to further extrapolate in analogy as a parallel to the initial example of concept verses tangible example and to proclaim the constant velocity of light as a special case of a general relative case in which it is a variable, leaving the (variable) velocity of light undefined and producing the existence of a weight in substitute for the value $\mathrm{C}$ ( the constant velocity of light) In this case, if one visualizes the described fulcrum, it also becomes obvious that in the activity of seeking such an object, the weight at both ends of the fulcrum is subject to change, as myth, a lessened social burden is assumed by the substitution of a physical weight for the constant velocity of light depending on a balance of social factors (in terms of its' myth) and physical factors, the historical and physical distance from the initial application of force by the mass, fact of' verification maybe prove less important as in the ordinary case, than aspects of beliefs; in terms of the influence of an unnatural physical weight on social life, an excess social weight can be potentially accrued depending on behavior that maybe assumed from the "myth of the theory of relativity" as belief in an entity that is ultimately the consequence of an actual physical weight, and can produce behavior, a mirroring behavior, as if a weight is actually imposed, to further enforce its' reality. In this case, a serious danger can 
emerge that is dependant on common understanding of science theory and the authority that it is granted.

In this case, a special case in mythology in which physical and not temporal distance exists from the distal end of the lever, scientific notions maintained distinct from the life lessons of myth and tale, the force of a hypothetical physical mass might be augmented if one lends justification from the theory of relativity to consider change at distal points (e.g. time travel) feasible; instead of lightening the weight/force to increase it by complying with it, necessarily reducing physical stability at both ends of the fulcrum, increasing mythological stability at the proximal end-affecting energy to mass (which need not necessarily reflect the quantity ' physical or biological weight') conversion from biological metabolic energy-a process of (excuse the pun) unen'light'enment, to make both heavy and dark.

Existing myth at their distal points, regardless of their physical or social validity are sacred to the process of enlightenment, should be maintained in a category isolated from fact, in this case constructed distinctly of 'mass' rather than weight in a gravitational field. Einsteins' appraisal of a potentially massless condition of energy might reflect in interpretation a perception that is the consequence of an existing serious breach in the path of enlightenment, demanding enlightenment, the theory of relativity never fully realized or understood.

The theory of relativity is based on observation of a location in space determined to be absent of mass, possessing only energy, containing a vacuum, and might be validly construed as threatening/frightening, mixed in together in religious belief with a fear of god; and imposes a conceptual lever of unquestionable potent common influence. It appears irresponsible, disastrous, to resolve public and common opinion into a separate category from scientific 
opinion; the only possible notion that is derivable for common relations from the theory of relativity is of a massless entity possessing energy, of a supernatural existence that is not held distinct from questionable scientific interpretation. Nearly infinitely distant from more important aspects of individual human niches, thew theory of relativity potentially bears an infinitely massive spiritual influence in which a related social responsibility already exists as well as an actual potential to unknowingly refer the entity of 'mass' in the theory to actual weight exists, depending on further scientific insight and understanding.

\section{NOTE}

About the theory of relativity, history, mathematics and science:

Parallel lines obviously do not intersect. Try to prove it on paper. You need lines that intersect in order to do that. The ancient Greeks were able to prove that if lines created angles of less than 90 degrees on the same side of a line that crossed them that they intersected, but left as postulate the converse that exact angles of 90 degrees in the same example did not intersect. If think about this, proof of the parallel postulate entails 'going beyond the plane of the paper, even then one is always left with proof onlyof the converse-without a defined point of intersection one is left with always left with concepts entailing the infinite which is only containable by definition in language; and the postulate that non intersecting (infinite) lines form 90 degree angles on the same side of some exisiting line that intersects them. The parallel postulate borders upon experience of the real world in terms of a relationship of the infinite to the eternal and can be extrapoated to refer to an ever-present logic to nature. The circumference of a circle, round and round and round might be viewed as infinite and is only captured with a notion of a (non infinite) radius with which it can be created. One can imagine a set of infinite radii but if he 
wishes to make them parallel to one another again returns to the parallel problem as postulation and an infinite set of potential radii to deal with no matter how many dimensions he wishes to consider. The recent proof of Pointecaires problem involving many dimensions, to show that if a figure is closed that there is some point that is equidistant to all surfaces in it, essentially that an intersection point exists for all closed spaces. The proof of the Pointcaire theorum required utiization of mathematical laws for the distribution of heat along surfacesand borders upon extending an understanding from mathematics to nature The real world however is considered to be open; a converse involving open surfaces remains unproven and we are always left with postulation. One might conclude that resolution hinges upon language definition as in the example of the circle of radii and circumferences, most important upon an understanding of language and mind themselves. It is coming to be understood that the facets of language may represent all there is to nature as a reflection of it-is composed of inverses and converses, equals and un-equals, additions and subtractions etc exactly as we conduct mathematics and sciencemost important both language and nature are open and composed of volumes and surfaces rather than lines, circles. and planes. In this presentation I focus on the geometry of the mobius strip into to illustrate the parallel problem and concepts in relativity. The mobius strip, a belt with a twist and rejoining in it, a fairly malleable structure physically, appears with little potential mathematical variation or complexity in analysis. The inside surface of the mobius strip goes round and round like the circumference of a circle, inside is continuous with outside. A single physical cut along the center of its' width results in two linked together untwisted strips. Other cuts divide the plane of the strip until they exceed the paper. Physical structure of the strip regarding its' width, viewed in this manner is limited by its' length at the center radius, provides a twist to the problem presented by Euclid in that it possess a radius (that extends into an infinite 
surface from which divides made at point, viewed at a tangential direction, 90 degrees from the (center line) line of the radius (also have no parallel counter parts due to th twist in the strip) result in two closed linked (untwisted)structures in which cuts that yield can yield closed contiguous loops are never parallel to one another anywhere in space because of the looped nature of the strip that is derived from the initial twist. All potential lines from either the original uncut surface or the cut resulting in loops, do not generate parallel lines though the loops are parallel to one another, never intersect, and arise from a single unique line that is parallel to itself only by definition. We thus have definable parallel and non parallel lines together in an open surface that can be visualized in many ways to enclose (open) volumes though the plane of the graphing paper, now possesses a twist, made as a new working radius aliken to the circle and its' circumference, leaves us still in the realm of postulation about angles and intersecting lines. Two (or more, an infinite number of ) mobius strips though can be visualized to be held parallel to one another as easily as two pieces of straight lumber can be nailed parallelily into place, and it might be asked if the pairs of loops(parallel to one another though appearing at an angle) from each parallel strip are necessarily parallel or not to one another. Individual and parallel mobius strips (e.g. the radii are made to fall into separate parallel planes) all loops involved might be defined as parallel to one another potentially, simultaneously that physical intersection can be described in illustration; but most important one can ask in a more general situation about representation of the world this way in terms of lines and planes, the faces of reality and its' contiguity. The world might be framed as a collection of lines and planes arising conceptually from a mobius geometry the describes contained rather than closed volumes, the indivisible link of derived loops not only figurative of a symmetry in nature (e.g. the plane of symmetry of the uracil molecule) but fitting closely to empirical scientific results pertaining to the genetic 
ordering of nature. Many molecules, especially those involved with in biological metabolisms, especially those involving the genetic material, DNA and its constituents have a mirror symmetry as does the carbon atom. It is possible to suggest that this symmetry is a parallel phenomenon, the divide from the radius of the mobius strip as a mirror; and that this divide longitudinally, along the radius, a measure of energy potential, provides along, with the width of mobius strips, the volumes and energies of open volumes as multiplication product.. In this sense, the contiguity of motion as one might perceive nature rather than as a set of still pictures, might be translated from the postulate of Euclid as a (non physically represented/existing) set of the set of (mobius) lines and planes that is reducible as a single unique line and unique parallel plane from which nature (energetic and order volumes) eminates as an experienced emerging uniqueness in which past does not converge, but is parallel with, conceptually, the present as a single contiguity.

Distance/ proximity when one experiments with nature should be measured in this way, as in the study of myths and their propagation rather than with respect to physical accessibility. The theory of relativity may have a ubiquitous appeal to become near in order to fill a void left open and laden with confusion, social and political conflict in the ascent of civilization.

It is also noteworthy to mention that mind/matter controversies appear to exist in two forms, as a conflict and as a paradox which unfortunately seem to endure in a mutually dependant fashion pending philosophical resolution of root paradox and have a pronounced negative influence on human behavior regarding values and the material aspects of existence. 


\section{References}

1) Einstein, Albert Physics and Reality Journal of the Franklin Institute 221:3 359-382 March 1986 